\title{
Absence of lysozyme (muramidase) in the intestinal Paneth cells of newborn infants with necrotising enterocolitis
}

H B Coutinho, H Carmona da Mota, V B Coutinho, T I Robalinho, A F Furtado, E Walker, G King, Y R Mahida, H F Sewell, D Wakelin
Table 1 Details of the patients studied

Aim-To determine immunocytochemically whether preterm and newborn infants with necrotising enterocolitis (NEC) show differences in numbers of lysozyme positive Paneth cells compared with normal controls, and to relate the findings to the possibility that lysozyme deficiency may facilitate the bacterial infections thought to be associated with this condition

Methods-Tissues from 10 infants with NEC and from 11 matched controls were sectioned and stained immunocytochemically for lysozyme. Differences in the numbers of Paneth cells and degree of lysozyme positivity in the tissues were assessed

Centro de Pesquisa Aggeu

Magalhaes/FIOCRUZ, Recife, Brazil

H B Coutinho

V B Coutinho

T I Robalinho

A F Furtado

Hospital Pediatrico, University of Coimbra, Portugal

C da Mota

Department of Pathology, University of Aberdeen, Scotland, UK

E Walker

G King

University of Nottingham, Nottingham, UK: Department of Medicine

Y R Mahida

Department of Immunology

H F Sewell

Department of Life Science

D Wakelin

Correspondence to: Professor H F Sewell, Department of Immunology, University of Nottingham Medical School, Queen's Medical Centre, Nottingham NG7 2UH, UK.

Accepted for publication 16 April 1998

\begin{tabular}{ll}
\hline Patient group & $\begin{array}{l}\text { Postconceptional age (weeks) at } \\
\text { diagnosis and/or operation }\end{array}$ \\
\hline Necrotising enterocolitis & \\
A & 30 \\
B & 40 \\
C & 32 \\
D & 40 \\
E & 40 \\
F & 40 \\
G & 33 \\
H & 30 \\
I & 31 \\
J & 32 \\
Intestinal atresia & \\
K & \\
L & 40 \\
M & 41 \\
N & 37 \\
O & 35 \\
P & 36 \\
Q & 39 \\
R & 36 \\
\hline
\end{tabular}
Rissues from NEC patients Paneth cells, whereas controls showed strong positive staining.

Conclusions-A deficiency or developmental defect in Paneth cells, resulting in an absence of lysozyme, may render the intestine more susceptible to bacterial infection, allowing organisms to adhere and translocate across the mucosa. Such enhancement of infection may contribute to the pathogenesis of NEC. (f Clin Pathol 1998;51:512-514)

Keywords: necrotising enterocolitis; Paneth cells; lysozyme; bacterial invasion

Necrotising enterocolitis (NEC) is the most frequent gastrointestinal emergency among preterm newborn infants, where it is associated with considerable mortality and morbidity. Recent data, for example, indicate that some 2000 to 4000 newborn infants in the USA are affected by NEC, resulting in approximately 1000 deaths a year. ${ }^{1}$ The pathogenesis of NEC remains incompletely understood, although bacterial agents may play an important role in a multifactorial process. ${ }^{12}$

The homeostasis and defence of the intestine against microorganisms, including those implicated in the aetiology of NEC, is provided by the adaptive and the innate immune systems. In preterm and term newborn infants the adaptive immune system is not completely differentiated ${ }^{3}$; plasma cells secreting IgA and IgM-immunoglobulins which are major components of the intestinal adaptive immune system-do not appear in significant numbers in the lamina propria until two or more weeks
Small intestinal specimens from another three post-term infants with intestinal obstruction were also used as controls.

after birth. ${ }^{4}$ As NEC usually appears in the first two weeks of life, it would seem that the absence of these immunoglobulins in the newborn infant does not play an important role in its pathogenesis. Attention is therefore focused on the innate immune system, of which the bactericidal enzyme lysozyme is considered a principal component. Lysozyme appears early in human ontogeny, and in the human small intestine is secreted by Paneth cells and Brunner's glands..$^{5-7}$

A significant decrease in the incidence of NEC in preterm and term newborn infants is reported in breast fed infants ${ }^{8}$ and it has been suggested that maternal lysozyme and immunoglobulins may have a protective role. Similar decreases in NEC are noted in newborn infants following antenatal or postnatal treatment with dexamethasone or betamethasone. ${ }^{910}$ It is relevant that the effectiveness of hydrocortisone acetate in inducing Paneth cell maturation has been shown in the marsupial Didelphis albiventris. ${ }^{11}$

These observations suggest that there may be abnormalities in the degree of Paneth cell differentiation - as evidenced by their production of lysozyme - in preterm and term newborn infants with NEC. Our results show for the first time a discrete deficiency within the intestinal innate immune system in NEC patients, characterised by the absence of lysozyme containing Paneth cells. 

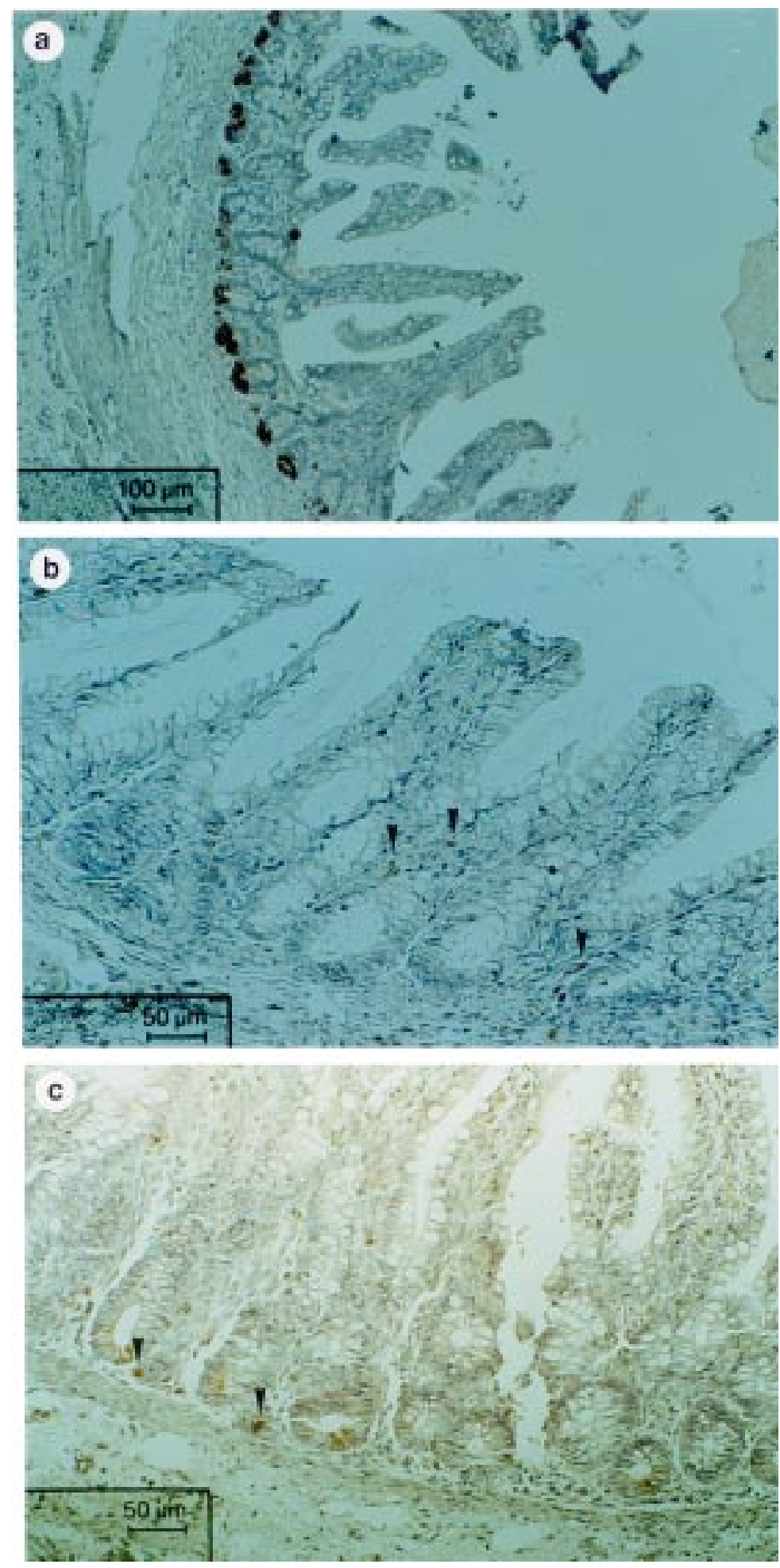

Figure 1 (a) Immunocytochemistry of small intestinal tissues from an intestinal atresic patient stained for lysozyme. Positive stain for lysozyme is present in the Paneth cells. (b) Immunocytochemistry of small intestinal tissues from an NEC patient stained for lysozyme. No lysozyme positive Paneth cells are visible. A few lysozyme positive macrophages can be seen (arrowed). (c) Immunocytochemistry of small intestinal tissues stained for lysozyme. In one NEC patient faintly stained lysozyme granules were present in some Paneth cells (arrowed).

\section{Methods}

PATIENTS AND TISSUES

Paraffin blocks of the small intestine mucosa, obtained during surgery from 10 newborn infants with NEC (including four term babies) and 11 newborn infants with neonatal obstruc- tion, including eight with intestinal atresia (four preterm) as controls, were taken from the archival files of the Hospital Pediatrico of Coimbra, Portugal, and the Department of Pathology, University of Aberdeen Royal Infirmary, Scotland, UK. The gestational age of the preterm infants varied from 30 to 36 weeks (table 1) and eight of them were operated on within the first week of life.

\section{IMMUNOCHEMISTRY}

In the cases studied, diagnosis had been established by clinical, radiological, surgical, and histopathological procedures. The sections studied were retrieved from tissues collected near the excision margins considered viable by the surgeons and remote from the necrotised area as defined by pathologists.

Sections were cut at 4 micron thickness, mounted on slides with 3-amino-propylmethoxylane, and dried at $56^{\circ} \mathrm{C}$ for 30 minutes. Immunostaining to demonstrate lysozyme was performed using a streptavidinbiotin complex horseradish peroxidase (sABC/ HRP) technique. Sections were dewaxed in xylene, and endogenous peroxidase blocked in a solution of $1.5 \%$ hydrogen peroxide in methanol. Optimal antigen retrieval-as detected using the primary rabbit antihuman lysozyme antibody (Dako A0099, Copenhagen, Denmark) - was obtained by digestion for five minutes in a solution of $0.1 \%$ trypsin prepared in distilled water containing $0.1 \%$ calcium chloride and adjusted to $\mathrm{pH} 7.8$ with $0.1 \mathrm{M}$ $\mathrm{NaOH}$. Sections were incubated in normal swine serum (Dako X0901) diluted 1:5 in TRIS buffered saline (TBS) for 10 minutes before incubation in the primary antihuman lysozyme antibody (diluted 1:10 000 in TBS) overnight at $4^{\circ} \mathrm{C}$. The remaining stages of the technique, including negative controls, were as previously described.

\section{Results}

All cases of intestinal atresia or obstruction used as controls showed strong positive staining for lysozyme in the cytoplasmic granules of Paneth cells found associated with the small intestine crypt epithelium (fig 1a). Lysozyme positive cells with the morphology of macrophages were also noted in the lamina propria.

In tissues from nine of 10 NEC patients, no lysozyme was detected immunocytochemically in Paneth cells; however, positive staining was detected in lamina propria macrophages (fig 1b). In a single NEC patient (33 weeks of gestation), rare Paneth cells were noted with faint lysozyme positive small cytoplasmic granules (fig 1c).

When the rabbit antihuman lysozyme antibody was substituted by buffer or an irrelevant primary layer antibody, no lysozyme was demonstrated in any section studied.

\section{Discussion}

Our results clearly show the absence of lysozyme containing Paneth cells in the intestinal mucosa of preterm and term newborn infants with NEC. At least two interpretations of this observation are possible. First, it could 
represent delayed maturation of lysozyme expressing Paneth cells in these patients. Second, the absence of these cells may be a secondary response to infection. We believe this latter explanation is unlikely because tissues from sites remote from the areas of severe necrosis/infection, and which were considered by surgeons and pathologists to represent normal tissues, also showed an absence of lysozyme positive Paneth cells.

Our data are more in keeping with a primary absence of mature lysozyme containing Paneth cells than a secondary response to infection or hypoxia leading to total inhibition of lysozyme transcription or translation. This is further supported by the detection of lysozyme containing macrophages within the same NEC tissue. The controls, which covered the same age range as the NEC cases, contained some surgical specimens from patients with torsion of the intestine, where secondary infection or ischaemic enteritis would have been likely. Nevertheless those control specimens did contain lysozyme positive Paneth cells. Additionally, the controls indicate that physiologically mature Paneth cells are normally present over the age range studied. Finally, we consider a postinfection response to be an unlikely explanation for the absence of lysozyme containing Paneth cells, as we have recently published a report on adults with severe intra-abdominal sepsis $^{12}$ in whom mature Paneth cells were still detectable (Coutinho HB; personal observations). Our findings suggest that the population with NEC that we studied may comprise individuals with delayed maturation of Paneth cells, leading to a lack of the potent antimicrobial agents such as lysozyme and defensins that are normally produced by these cells. ${ }^{13-16}$ The absence of these defensive factors could facilitate bacterial survival, adherence, and mucosal translocation, all of which are thought to be involved in the pathogenesis of NEC.

The report of a decreased incidence of NEC in breast fed preterm infants ${ }^{8}$ is in accord with our present study as it is known that large quantities of lysozyme, together with protective maternal antibodies, are present in milk. Experimental models have documented the induction of Paneth cell differentiation by parenteral administration of glucocorticoid, resulting in the cells acquiring their characteristic granularity together with the presence of lysozyme. ${ }^{11}$ Interestingly, controlled clinical trials using prenatal and postnatal administration of steroids have resulted in significant decreases in NEC incidence. ${ }^{9}{ }^{10}$ Our studies reported here give a scientific rationale for the results obtained using steroids in both the experimental and clinical studies. Consideration could be given to the use of other inducers of Paneth cell maturation/differentiation as prophylaxis against NEC.

We are well aware that our study population - which included no very low birthweight preterm babies and a high proportion of term infants who presented with early onset of manifestations of NEC-may not be representative of the NEC population as a whole. Nevertheless we believe the findings raise some possibilities concerning the selection of a subpopulation at risk of developing the disease. For instance, the measurement of lysozyme in meconium may prove to be a helpful predictor for defining at risk groups.

Finally, the absence of lysozyme may relate directly to NEC pathogenesis or it may represent an indirect but parallel marker of the absence of other Paneth cell products, which themselves may be more directly related to the prevention of NEC. However, if lysozyme is indeed central to the prevention of NEC it raises the possibility of additional means of controlling this disease. Clearly, continued and increased use of breast milk should be encouraged to prevent NEC. However, when breast milk is not available, the possibility of using purified or recombinant derived lysozyme for NEC management, either alone or added to an enriched formula, should be considered.

We are indebted to Dr Fátima Sarabando, pathologist, and Dr Francelina Lopes, surgeon, University of Coimbra, Portugal, and Dr Elizabeth Gray, paediatric pathologist, University of and Dr Elizabeth Gray, paediatric pathologist, University of
Aberdeen, for NEC and control specimens. We thank Miss A M Aberdeen, for NEC and control specimens. We thank Miss A M
S Amorim for secretarial assistance and Mr R N Pimentel for $\mathrm{S}$ Amorim for secretarial assistance and $\mathrm{Mr} \mathrm{R} \mathrm{N}$ Pimentel for
photography. This work was supported by grants from The British Council, FACEPE and CNPq (Brazil), JNICT (Portugal), and the EEC.

1 Neu J. Necrotizing enterocolitis: the search for a unifying pathogenic theory leading to prevention. Pediatr Clin North Am 1996;43:409-32.

2 Israel EJ. Neonatal necrotizing enterocolitis, a disease of the immature intestinal mucosal barrier. Acta Paediatr Suppl 1994;396:27-32.

3 MacDonald T, Spencer JM. Ontogeny of the associated lymphoid system in man. Acta Paediatr Suppl 1994;395:35.

4 Perkkio M, Savilahti E. Time of appearance of immunoglobulin-containing cells in the mucosa of neonaimmunoglobulin-containing cells in the

5 Brandtzaeg P, Baklein K, Bjerke K, et al. Nature of the human gastrointestinal immune system. In: Miller K, Nicklin S, eds. Immunology of the gastrointestinal tract. Boca Raton, Florida: CRC Press, 1987:1-86.

6 Caruso RA, LaSpada F, Casablanca G, et al. Lysozyme and mucins in gastric adenomas. $\mathcal{F}$ Clin Pathol 1989;42:827-33.

7 Coutinho HB, Robalinho TI, Coutinho VB, et al. Immunocytochemical demonstration that human duodenal Brunner's glands may participate in the intestinal defence. $f$ Anat 1996;189:193-7.

8 Lucas A, Cole TJ. Breast milk and neonatal necrotizing enterocolitis in premature infants. Lancet 1990;336:151923.

9 Bauer CR, Morrison JC, Poole KW, et al. A decreased incidence of necrotizing enterocolitis after prenatal glucocorticoid therapy. Pediatrics 1984;73:682-8.

0 Halac E, Halac J, Begue EF, et al. Prenatal and postnatal corticosteroid therapy to prevent necrotizing enterocolitis: a controlled trial. F Pediatr 1990;117:132-8.

11 Coutinho VB, Coutinho HB, Coutinho EM. Effects of hydrocortisone acetate treatment on the small intestine of the lactent marsupial Didelphis albiventris. Anat Anz fena 1991;172:213-21.

12 Coutinho HB, Robalinho TI, Coutinho VB, et al. Intraabdominal sepsis: an immunocytochemical study of the abdominal intestine mucosa. 7 Clin Pathol 1997 50:294-8.

$13 \mathrm{McNabb} P$, Tomasi TB. Host defence mechanisms at mucosal structures. Annu Rev Microbiol 1981;35:477-96.

14 Elmes ME, Stanton MR, Howells CHL, et al. Relation between the mucosal flora and Paneth cell population of human jejunum and ileum. $\mathcal{F}$ Clin Pathol 1984;37:1268-71.

15 Selsted ME, Miller SJ, Henshen AH, et al. Enteric defensins: antibiotic peptide components of infestinal host defense. $\mathcal{F}$ Cell Biol 1992;118:929-36.

16 Ouellete AJ. Paneth cells and innate immunity in the crypt micro-environment. Gastroenterology 1997;113:1779-84. 